\title{
FUNCTIONAL DIFFERENTIAL EQUATIONS AND ONE-DIMENSIONAL DISTORTIONLESS PROPAGATION
}

\author{
VLADIMIR RĂSVAN
}

\begin{abstract}
One-dimensional propagation phenomena of physics and technology are described by boundary value problems for hyperbolic partial differential equations in one space dimension. Since the paper of J. Bernoulli published in 1728, functional equations are usually associated with such problems, the best known in the last decades being the neutral differential equations with deviated arguments. The distortionless propagation corresponds to the case of the equations with pointwise (lumped) time delays. The present paper will consider some applications of these equations in nuclear and power engineering, engineering mechanics, hydraulics. The basic model validation steps (basic theory, invariant sets, inherent stability) are discussed.
\end{abstract}

\section{Basic examples}

According to a well known pioneering reference 14 the first reference about a differential equation with deviating argument goes back to the XVIIIth century and is due to Joh an n B e r noulli [3]. As the (Latin) title of the paper shows, he considered a weighted stretched vibrating cord with distributed masses on it. Finally he was led to the equation

$$
\dot{y}=y(t-1) \text {. }
$$

For our goal it is important to note that this equation arose from a problem of partial differential equations moreover, of hyperbolic type, even if the association appeared to be mistaken. The fact that functional equations may be associated to various boundary value problems for PDE became an elementary truth in the

2000 Mathematics Subject Classification: 35L20, 34K40, 34K35.

Keywords: hyperbolic partial differential equations, boundary value problems, neutral functional equations, lumped time delays.

This work has been partly supported from the Project PCE ID-95 of the Romanian Council for the Research in Higher Education. 


\section{VLADIMIR RĂSVAN}

XXth century or even earlier. From the long list of references we pick up a rather general situation considered by K. L. Cooke [7]

$$
\begin{aligned}
& \frac{\partial u_{1}}{\partial t}+\tau_{1}(\lambda, t) \frac{\partial u_{1}}{\partial \lambda}=\Phi_{1}(\lambda, t), \\
& \frac{\partial u_{2}}{\partial t}+\tau_{2}(\lambda, t) \frac{\partial u_{2}}{\partial \lambda}=\Phi_{2}(\lambda, t), \quad 0 \leq \lambda \leq 1, \quad t \geq t_{0},
\end{aligned}
$$

with the boundary conditions described by ordinary differential equations

$$
\begin{aligned}
& \sum_{k=0}^{m}\left[a_{k 1}(t) \frac{d^{k}}{\mathrm{~d} t^{k}} u_{1}(0, t)+a_{k 2}(t) \frac{d^{k}}{\mathrm{~d} t^{k}} u_{2}(0, t)\right]=f_{1}(t), \\
& \sum_{k=0}^{m}\left[b_{k 1}(t) \frac{d^{k}}{\mathrm{~d} t^{k}} u_{1}(1, t)+b_{k 2}(t) \frac{d^{k}}{\mathrm{~d} t^{k}} u_{2}(1, t)\right]=f_{2}(t)
\end{aligned}
$$

with the initial conditions:

$$
u_{1}\left(\lambda, t_{0}\right)=\omega_{1}(\lambda), \quad u_{2}\left(\lambda, t_{0}\right)=\omega_{2}(\lambda), \quad 0 \leq \lambda \leq 1,
$$

and under the assumption that $\tau_{1}(\lambda, t)>0, \tau_{2}(\lambda, t)<0$ in the considered domain (what gives the hyperbolic character to the system): $u_{1}(\lambda, t)$ is the so-called forward wave and $u_{2}(\lambda, t)$ - the backward wave. Mathematically speaking, these waves represent the Riemann invariants of the problem. Consider the system of the characteristics

$$
\frac{\mathrm{d} t}{\mathrm{~d} \lambda}=\frac{1}{\tau_{i}(\lambda, t)}, \quad i=1,2, \quad 0 \leq \lambda \leq 1, \quad t \geq t_{0}
$$

Let $t_{1}(\lambda ; 0, t)$ be an increasing characteristic curve (verifying the equation associated to $\tau_{1}(\lambda, t)>0$, i.e., to the forward wave) starting from the boundary point $(0, t)$. Since $\tau_{1}(\lambda, t)$ is continuous and $(0,1)$ is a bounded interval, $t_{1}(\lambda, 0, t)$ is continuable on $(0,1)$ and we may define $T_{1}(t)$ - the forward propagation timeas $T_{1}(t)=t_{1}(1 ; 0, t)-t($ see Fig:1).

If we consider the forward wave along the increasing characteristic

$$
\phi_{1}(\lambda)=u_{1}\left(\lambda, t_{1}(\lambda ; 0, t)\right),
$$

make use of the first equation of (10) and integrate with respect to $\lambda$, we obtain for each fixed $t$

$$
\phi_{1}(\lambda)=\phi_{1}(0)+\int_{0}^{\lambda} \frac{\Phi_{1}\left(\sigma, t_{1}(\sigma ; 0, t)\right)}{\tau_{1}\left(\sigma, t_{1}(\sigma ; 0, t)\right)} \mathrm{d} \sigma
$$

which reads

$$
\phi_{1}(1)=u_{1}\left(1, t_{1}(1 ; 0, t)\right)=u_{1}\left(1, t+T_{1}(t)\right)=u_{1}(0, t)+\int_{0}^{1} \frac{\Phi_{1}\left(\sigma, t_{1}(\sigma ; 0, t)\right)}{\tau_{1}\left(\sigma, t_{1}(\sigma ; 0, t)\right)} \mathrm{d} \sigma,
$$




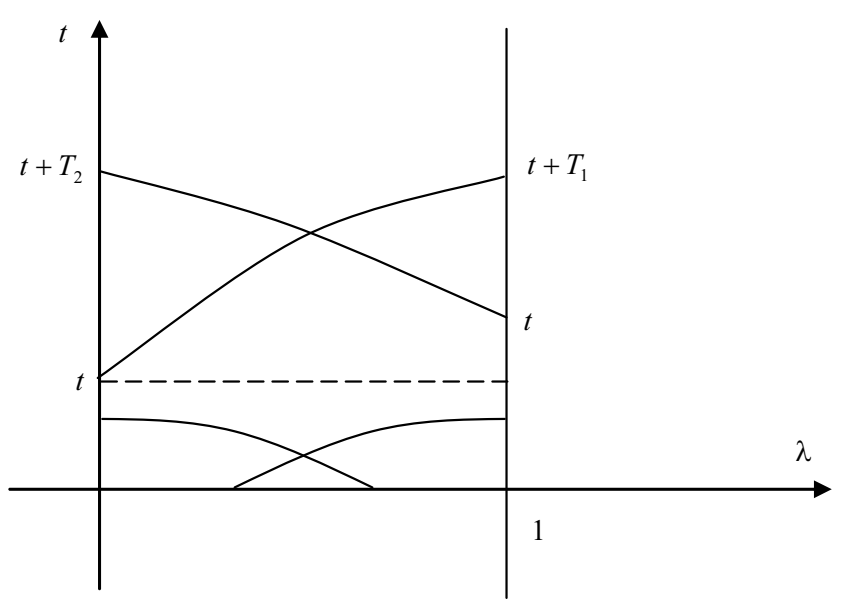

FiguRE 1. Characteristic curves.

in fact a functional relation between the boundary values of the forward wave.

Similarly, let $t_{2}(\lambda ; 1, t)$ be a decreasing characteristic curve: we may define the backward propagation time $T_{2}(t)$ as $t_{2}(0 ; 1, t)-t$ and obtain the functional relation between the boundary values of the backward wave

$$
u_{2}\left(0, t+T_{2}(t)\right)=u_{2}(1, t)+\int_{0}^{1} \frac{\Phi_{2}\left(\sigma, t_{2}(\sigma ; 1, t)\right)}{\tau_{2}\left(\sigma, t_{2}(\sigma ; 1, t)\right)} \mathrm{d} \sigma .
$$

Denoting for the simplicity of equation writing

$$
\begin{aligned}
y_{1}(t) & =u_{1}(1, t), & y_{2}(t) & =u_{2}(0, t), \\
\psi_{1}(t) & =\int_{0}^{1} \frac{\Phi_{1}\left(\sigma, t_{1}(\sigma ; 0, t)\right)}{\tau_{1}\left(\sigma, t_{1}(\sigma ; 0, t)\right)} \mathrm{d} \sigma, & \psi_{2}(t) & =\int_{0}^{1} \frac{\Phi_{2}\left(\sigma, t_{2}(\sigma ; 1, t)\right)}{\tau_{2}\left(\sigma, t_{2}(\sigma ; 1, t)\right)} \mathrm{d} \sigma .
\end{aligned}
$$

and substituting in the boundary conditions (2), the following system of differential equations with deviating argument is obtained:

$$
\begin{aligned}
& \sum_{k=0}^{m}\left[a_{k 1}(t) \frac{d^{k}}{\mathrm{~d} t^{k}} y_{1}\left(t+T_{1}(t)\right)+a_{k 2}(t) \frac{d^{k}}{\mathrm{~d} t^{k}} y_{2}(t)\right]=f_{1}(t)+\sum_{k=0}^{m} a_{k 1}(t) \frac{d^{k}}{\mathrm{~d} t^{k}} \psi_{1}(t), \\
& \sum_{k=0}^{m}\left[b_{k 1}(t) \frac{d^{k}}{\mathrm{~d} t^{k}} y_{1}(t)+b_{k 2}(t) \frac{d^{k}}{\mathrm{~d} t^{k}} y_{2}\left(t+T_{2}(t)\right)\right]=f_{2}(t)+\sum_{k=0}^{m} b_{k 1}(t) \frac{d^{k}}{\mathrm{~d} t^{k}} \psi_{2}(t) .
\end{aligned}
$$




\section{VLADIMIR RĂSVAN}

In fact, if $\left(u_{1}(\lambda, t), u_{2}(\lambda, t)\right)$ is a solution of (11)-(3), then $\left(y_{1}(t), y_{2}(t)\right)$ is a solution of the system (10); the initial conditions of (10) can also be determined from the initial conditions of the mixed problem. The converse is also true: using the representation formulae

$$
\begin{aligned}
& u_{1}(\lambda, t)=y_{1}\left(t_{1}(1 ; \lambda, t)\right)-\int_{\lambda}^{1} \frac{\Phi_{1}\left(\sigma, t_{1}(\sigma ; \lambda, t)\right)}{\tau_{1}\left(\sigma, t_{1}(\sigma ; \lambda, t)\right)} \mathrm{d} \sigma, \\
& u_{2}(\lambda, t)=y_{2}\left(t_{2}(1 ; \lambda, t)\right)+\int_{0}^{\lambda} \frac{\Phi_{2}\left(\sigma, t_{2}(\sigma ; \lambda, t)\right)}{\tau_{2}\left(\sigma, t_{2}(\sigma ; \lambda, t)\right)} \mathrm{d} \sigma
\end{aligned}
$$

it is possible to fully describe the solution of the mixed problem by the solution of the mixed problem (11)-(3) using the solution of the Cauchy problem for (10). This may be stated as a theorem as follows

Theorem 1. Consider the mixed initial boundary value problem defined by (11)-(3) under the above assumptions. If $\left(u_{1}(\lambda, t), u_{2}(\lambda, t)\right)$ is a solution of it, then $\left(y_{1}(t), y_{2}(t)\right)$ defined by (9) is a solution of the system (10) with suitable initial conditions. Conversely, if $\left(y_{1}(t), y_{2}(t)\right)$ is a solution of the system (10) with some initial conditions, then $\left(u_{1}(\lambda, t), u_{2}(\lambda, t)\right)$ defined by the representation formulae (11) is a solution of (11)-(3) with the initial conditions (3) deduced accordingly from the initial conditions of $\left(y_{1}(t), y_{2}(t)\right)$.

Two additional remarks may be formulated concerning the above one to one correspondence. The first one is that it represents a natural way of introducing all types of differential equations with deviated argument; indeed, following [7], introduce the following integers

$$
\begin{gathered}
0<L_{1}=\max \left\{k: a_{k 1}(t) \not \equiv 0\right\}, \quad 0<L_{2}=\max \left\{k: b_{k 2}(t) \not \equiv 0\right\}, \\
0<K_{1}=\max \left\{k: b_{k 1}(t) \not \equiv 0\right\}, \quad 0<K_{2}=\max \left\{k: a_{k 2}(t) \not \equiv 0\right\}, \\
M=L_{1}+L_{2}-\left(K_{1}+K_{2}\right) .
\end{gathered}
$$

The boundary conditions (21) are said to be of retarded, neutral or advanced type if $M>0, M=$ ) or $M<0$ accordingly; this terminology is analogous to that familiar in the theory of functional differential equations; also the evolution in time of the smoothness of the solutions is in accordance with that known in the classification of the nonlinear functional differential equations (the so-called Kamenskii classification of 1958, see [10]). In particular, if $M<0$ (the advanced type case) there is a loss of smoothness at each step and, if the initial conditions are not analytic or at least $\mathcal{C}^{\infty}$, the solution exists up to a certain natural boundary; also smoothness remains unchanged in the neutral case $(M=0)$ or is even increasing (if $M>0$ ). 


\section{ONE-DIMENSIONAL DISTORTIONLESS PROPAGATION}

The second remark is that (10) is a system with pointwise argument deviations and if these deviations were constants, the case would correspond to the so-called distortionless propagation. As it will be shown next, distortionless propagation follows from lossless and decoupling of the basic waves propagation equation.

\section{Distortionless propagation}

As pointed out in [4], distortionless "signalling" is of fundamental importance in applied science; the simplest case arises in the equation of the vibrating string

$$
c^{2} \frac{\partial^{2} v}{\partial \lambda^{2}}=\frac{\partial^{2} v}{\partial t^{2}}
$$

whose general solution is

$$
v(\lambda, t)=\phi\left(t-\frac{\lambda}{c}\right)+\psi\left(t+\frac{\lambda}{c}\right)
$$

with $\phi, \psi$ arbitrary functions : $\phi(t-\lambda / c)$ is a wave travelling "to the right" with velocity $c$ - the forward wave; this wave is transmitted to a distant point $\lambda$ without distortion but with a time delay $\tau=\lambda / c$.

The equation for spherical waves in $n$ dimensions is

$$
c^{2}\left(\frac{\partial^{2} v}{\partial r^{2}}+\frac{n-1}{r} \frac{\partial v}{\partial r}\right)=\frac{\partial^{2} v}{\partial t^{2}}
$$

and if $n=3$ the general solution is

$$
v(r, t)=\frac{1}{r}\left(\phi\left(t-\frac{r}{c}\right)+\psi\left(t+\frac{r}{c}\right)\right) .
$$

Again we obtain a distortionless transmission but with attenuation $r^{-1}$. As pointed out in [9], it is a very happy circumstance that we live in a three dimensional space because signals can be broadcasted without distortion only if $n=1$ or $n=3$.

A. A most common transmission system is the electrical transmission line that can be, in the general case, inhomogeneous and dissipative; its equations (the "telegraph equations") are as follows

$$
-\frac{\partial v}{\partial \lambda}=r(\lambda) i(\lambda, t)+l(\lambda) \frac{\partial i}{\partial t}, \quad-\frac{\partial i}{\partial \lambda}=g(\lambda) v(\lambda, t)+c(\lambda) \frac{\partial v}{\partial t},
$$

where the two waves are no longer separated as in (1). In fact these are considered as the general telegraph equations and the wave equations are one of their special cases. 


\section{VLADIMIR RĂSVAN}

It is said that the transmission line is distortionless if there is a solution of the form

$$
v(\lambda, t)=f(\lambda) \phi(t-\tau(\lambda)), \quad v(0, t)=\phi(t) .
$$

Here the function $f(\cdot)$ is called attenuation and $\tau(\cdot)$ is the propagation delay; they have to be independent of $\phi$ and also $f(0)=1, \tau(0)=0$. Under the natural assumptions of physical nature : $c(\lambda)>0, l(\lambda)>0, r(\lambda) \geq 0, g(\lambda) \geq 0$, there are required conditions on these line parameters to obtain a distortionless transmission. Several such cases are discussed in [4] among which we cite the case of the resistive line - when the ratio $v(\lambda, t) / i(\lambda, t)$ is independent of $t$ - or the case of both voltage and current distortionless line - when both $v(\lambda, t)$ and $i(\lambda, t)$ have the form (18). The conditions obtained in (op. cit) are expressed in the language of line's parameters and may be fulfilled technologically; there exist many other conditions of distortionless propagation (op. cit) but we shall leave them aside and consider the problem from a more mathematical point of view.

B. System (17) is a symmetric system in the sense of Friedrichs and we have the possibility to introduce the Riemann invariants by

$$
u^{ \pm}(\lambda, t)=v(\lambda, t) \pm a(\lambda) i(\lambda, t)
$$

or by the converse equalities

$$
\begin{aligned}
& v(\lambda, t)=\frac{1}{2}\left[u^{+}(\lambda, t)+u^{-}(\lambda, t)\right] \\
& i(\lambda, t)=\frac{1}{2 a(\lambda)}\left[u^{+}(\lambda, t)-u^{-}(\lambda, t)\right] .
\end{aligned}
$$

A rather straightforward manipulation will change them to the following equations

$$
\begin{aligned}
-\frac{\partial u^{+}}{\partial \lambda}= & \sqrt{l(\lambda) c(\lambda)} \frac{\partial u^{+}}{\partial t} \\
& +\frac{1}{2}\left(a(\lambda) g(\lambda)+\frac{r(\lambda)-a^{\prime}(\lambda)}{a(\lambda)}\right) u^{+}(\lambda, t) \\
& +\frac{1}{2}\left(a(\lambda) g(\lambda)-\frac{r(\lambda)-a^{\prime}(\lambda)}{a(\lambda)}\right) u^{-}(\lambda, t), \\
-\frac{\partial u^{-}}{\partial \lambda}= & -\sqrt{l(\lambda) c(\lambda)} \frac{\partial u^{-}}{\partial t} \\
& -\frac{1}{2}\left(a(\lambda) g(\lambda)-\frac{r(\lambda)+a^{\prime}(\lambda)}{a(\lambda)}\right) u^{+}(\lambda, t) \\
& -\frac{1}{2}\left(a(\lambda) g(\lambda)+\frac{r(\lambda)+a^{\prime}(\lambda)}{a(\lambda)}\right) u^{-}(\lambda, t),
\end{aligned}
$$




\section{ONE-DIMENSIONAL DISTORTIONLESS PROPAGATION}

where $a(\lambda)=\sqrt{l(\lambda) / c(\lambda)}$. If we impose the distortionless propagation for the forward wave $u^{+}$, this will mean the first equation decoupled from the second one; therefore $a(\lambda)$ has to be chosen as satisfying the Riccati equation below

$$
a^{\prime}(\lambda)=r(\lambda)-g(\lambda) a^{2}(\lambda)
$$

which is to be found as one of the necessary conditions for any distortionless propagation [4]. Equations (21) become

$$
\begin{aligned}
-\frac{\partial u^{+}}{\partial \lambda}= & \sqrt{l(\lambda) c(\lambda)} \frac{\partial u^{+}}{\partial t}+g(\lambda) \sqrt{\frac{l(\lambda)}{c(\lambda)}} u^{+}(\lambda, t), \\
-\frac{\partial u^{-}}{\partial \lambda}= & -\sqrt{l(\lambda) c(\lambda)} \frac{\partial u^{-}}{\partial t}-r(\lambda) \sqrt{c(\lambda) / l(\lambda)} u^{-}(\lambda, t) \\
& +(r(\lambda) \sqrt{c(\lambda) / l(\lambda)}-g(\lambda) \sqrt{l(\lambda) / c(\lambda)}) u^{+}(\lambda, t) .
\end{aligned}
$$

Putting

$$
\begin{aligned}
& w^{+}(\lambda, t)=\exp \left(\int_{0}^{\lambda} g(\sigma) a(\sigma) \mathrm{d} \sigma\right) u^{+}(\lambda, t), \\
& w^{-}(\lambda, t)=\exp \left(-\int_{\lambda}^{1}(r(\sigma) / a(\sigma)) \mathrm{d} \sigma\right) u^{-}(\lambda, t)
\end{aligned}
$$

will transform system (23) to

$$
\begin{aligned}
-\frac{\partial w^{+}}{\partial \lambda} & =\sqrt{l(\lambda) c(\lambda)} \frac{\partial w^{+}}{\partial t}, \\
-\frac{\partial w^{-}}{\partial \lambda} & =-\sqrt{l(\lambda) c(\lambda)} \frac{\partial w^{-}}{\partial t}+\beta(\lambda) w^{+}(\lambda, t),
\end{aligned}
$$

where

$$
\beta(\lambda)=\left(\frac{r(\lambda)}{a(\lambda)}-g(\lambda) a(\lambda)\right) \exp \left(-\int_{0}^{\lambda} g(\sigma) a(\sigma) \mathrm{d} \sigma-\int_{\lambda}^{1}(r(\sigma) / a(\sigma)) \mathrm{d} \sigma\right) .
$$

If the following additional matching condition (nothing more but the generalized to nonuniform lines Heaviside condition) is met

$$
r(\lambda) \sqrt{\frac{c(\lambda)}{l(\lambda)}}=g(\lambda) \sqrt{\frac{l(\lambda)}{c(\lambda)}} \quad \text { almost everywhere on }(0,1),
$$

then $\beta(\lambda)=0$ a.e. on $(0,1)$ and the two Riemann invariants (the "waves") are decoupled as in the previous section, hence the propagation is distortionless. We do not insist here on the transformation of the boundary conditions since this will appear quite clear in the applications that follow. 


\section{VLADIMIR RĂSVAN}

\section{Two applications}

A. The first application-feedback control of an overhead crane - is considered as benchmark in Control Theory, even if it is considered from rather different viewpoints. For instance, its nonlinear model with lumped parameters, i.e., described by ordinary differential equations [1] was used for the application of recent nonlinear control techniques [11, 12].

A more recent model [2] takes into account the distributed elasticity of crane's pendulum rod and is as follows

$$
\begin{aligned}
\frac{\partial^{2} y}{\partial t^{2}}-\frac{\partial}{\partial \lambda}\left(a(\lambda) \frac{\partial y}{\partial \lambda}\right) & =0, \quad t>0,0 \leq \lambda \leq L \\
\frac{\partial y}{\partial \lambda}(0, t) & =0, \quad y(L, t)=x_{p}, \quad \ddot{x}_{p}=\gamma_{0} a(L) \frac{\partial y}{\partial \lambda}(L, t)+u(t)
\end{aligned}
$$

which is much alike to the general structures considered previously: while the boundary conditions are quite standard, they are controlled by a system of ordinary differential equations, itself controlled by the boundary values. We have in fact a standard "indirect" boundary control problem; the term "indirect" arises from the classical Control Theory and its meaning is that the control signal $u(t)$ is applied at the boundary of the distributed parameter area through a lumped parameter controller having its own dynamics.

A first change of functions will give the form (17) to the partial differential equations and will modify the boundary conditions accordingly

$$
v(\lambda, t) \equiv \frac{\partial y}{\partial t}(\lambda, t), \quad w(\lambda, t) \equiv a(\lambda) \frac{\partial y}{\partial \lambda}(\lambda, t)
$$

and, therefore

$$
\begin{gathered}
\frac{\partial v}{\partial t}-\frac{\partial w}{\partial \lambda}, \quad \frac{\partial w}{\partial t}-a(\lambda) \frac{\partial v}{\partial \lambda}=0 \\
w(0, t)=0, \quad v(L, t)=\dot{x}_{p}, \quad \ddot{x}_{p}=\gamma_{0} w(L, t)+u(t) .
\end{gathered}
$$

Next, we introduce the forward and backward waves - the Riemann invariants

$$
u^{ \pm}(\lambda, t)=w(\lambda, t) \mp \sqrt{a(\lambda)} v(\lambda, t)
$$

to obtain their equations

$$
\frac{\partial u^{ \pm}}{\partial t} \pm \sqrt{a(\lambda)} \frac{\partial u^{ \pm}}{\partial \lambda}=\frac{a^{\prime}(\lambda)}{4 \sqrt{a(\lambda)}}\left(u^{+}(\lambda, t)-u^{-}(\lambda, t)\right) .
$$

Here the distortionless propagation condition is $a^{\prime}(\lambda)=0$ a.e., i.e., $a(\lambda)$ has to be, e.g., piecewise constant. We may deduce that in this case distortions are introduced by a certain degree of non-uniform elasticity. 


\section{ONE-DIMENSIONAL DISTORTIONLESS PROPAGATION}

Assuming that we are in the distortionless case (which is also lossless), we shall consider the two families of characteristics

$$
\begin{gathered}
\frac{\mathrm{d} t}{\mathrm{~d} \lambda}= \pm \frac{1}{\sqrt{a(\lambda)}}, \\
t^{+}(\lambda ; 0, t)=t+\int_{0}^{\lambda} \frac{\mathrm{d} \sigma}{\sqrt{a(\sigma)}}, \quad t^{-}(\lambda ; L, t)=t+\int_{\lambda}^{L} \frac{\mathrm{d} \sigma}{\sqrt{a(\sigma)}}
\end{gathered}
$$

and the time delay propagation

$$
\tau=\int_{0}^{L} \frac{\mathrm{d} \sigma}{\sqrt{a(\sigma)}}
$$

Integration of the Riemann invariants along the characteristics will give

$$
u^{+}(0, t)=u^{+}(L, t+\tau), \quad u^{-}(L, t)=u^{-}(0, t+\tau) .
$$

If these equalities are introduced in the boundary conditions, with suitable notations and after some simple manipulation the following system of functional differential equations is associated

$$
\begin{aligned}
\ddot{x}_{p}+\frac{\gamma_{0}}{\sqrt{a(L)}} \dot{x}_{p} & =2 \gamma_{0} \eta(t)+u(t), \\
\eta(t)+\eta(t-2 \tau) & =\frac{1}{\sqrt{a(L)}} \dot{x}_{p}
\end{aligned}
$$

which is a Cartesian product of an ODE and a shift equation - known to be equivalent to a neutral FDE [18]. Also we may introduce the variable $v_{p}=\dot{x}_{p}$ and then eliminate $v_{p}$ in (35) to obtain

$$
\frac{\mathrm{d}}{\mathrm{d} t}(\eta(t)+\eta(t-2 \tau))+\frac{2 \gamma_{0}}{\sqrt{a(L)}} \eta(t-2 \tau)=u(t)
$$

which is obviously a neutral FDE.

B. Another application involving distortionless propagation is the dynamics of circulating fuel nuclear reactor. This technology is again an object of detailed analysis (after an interruption of 20-25 years) see, e.g., [5]. One of the standard models is that of [13, where the delayed neutrons are "circulating" as a distortionless propagation of a fluid wave

$$
\left\{\begin{array}{l}
\frac{\mathrm{d}}{\mathrm{d} t} n(t)=\rho n(t)+\sum_{i=1}^{m} \beta_{i}\left(\bar{c}_{i}(t)-n(t)\right), \quad \bar{c}_{i}(t)=\int_{0}^{h} \phi(\eta) c_{i}(\eta, t) \mathrm{d} \eta \\
\frac{\partial c_{i}}{\partial t}+\frac{\partial c_{i}}{\partial \eta}+\sigma_{i} c_{i}=\sigma_{i} \phi(\eta) n(t), \quad c_{i}(0, t)=c_{i}(h, t), \quad t \geq t_{0} \\
c_{i}\left(\eta, t_{0}\right)=q_{i}^{0}(\eta), \quad i=\overline{1, m}, \quad n\left(t_{0}\right)=n_{0}, \quad 0 \leq \eta \leq h .
\end{array}\right.
$$




\section{VLADIMIR RĂSVAN}

Let us mention that the boundary conditions are here of periodic type. The partial differential equations are already decoupled what ensures distortionless propagation; moreover, only a single family of characteristics is present here, hence only forward waves are defining the solution. The same approach as previously will associate the following system of FDE

$$
\begin{aligned}
\frac{\mathrm{d} n}{\mathrm{~d} t}= & \left(\rho-\sum_{1}^{m} \beta_{i}\right) n(t)+\sum_{i=1}^{m} \beta_{i} \sigma_{i} \int_{-h}^{0} e^{\lambda \sigma_{i}}\left(\int_{-\lambda}^{h} \phi(\lambda) \phi(\eta+\lambda) \mathrm{d} \eta\right) n(t+\lambda) \mathrm{d} \lambda \\
& +\sum_{i=1}^{m} \beta_{i} \int_{-h}^{0} e^{\eta \sigma_{i}} \phi(-\eta) q_{i}(t+\eta) \mathrm{d} \eta, \\
q_{i}(t+h)= & e^{-h \sigma_{i}}\left[q_{i}(t)+\sigma_{i} \int_{0}^{h} e^{\lambda \sigma_{i}} \phi(\lambda) n(t+\lambda) \mathrm{d} \lambda\right] .
\end{aligned}
$$

Observe that the equations for $q_{i}$ contain a lumped time delay accounting for distortionless propagation while the variable $n$ displays a distributed delay due to the fact that it enters the partial differential equations. The difference equations for $q_{i}(t)$ show again a system of FDE of neutral type.

\section{Various problems and solutions}

Previous considerations showed a technique of associating FDE of neutral type (mainly) to IBVP for some hyperbolic PDE. The so-called distortionless propagation is associated to neutral equations with lumped delays. Such functional equations may be considered as representing self contained mathematical objects for which standard problems may be formulated and solved [16, 17]. If we consider, for instance, the linear system

$$
\left\{\begin{array}{l}
\dot{x}^{1}(t)=A_{0} x^{1}(t)+A_{1} x^{2}(t-\tau), \\
x^{2}(t)=A_{2} x^{1}(t)+A_{3} x^{2}(t-\tau),
\end{array}\right.
$$

a Cauchy problem and exponential stability may be considered. System (35) with $u(t) \equiv 0$ belongs to this class. Its characteristic equation is

$$
p(s) \equiv s\left[\left(1+\mathrm{e}^{-2 s \tau}\right) s+\gamma_{1} \mathrm{e}^{-2 s \tau}\right]=0, \quad \gamma_{1}=\frac{2 \gamma_{0}}{\sqrt{a(L)}}
$$

and obviously requires stabilization since it has at least a zero root. It is not obvious if (35) is stabilizable, in any case we need to know more about the roots of the other factor which coincide with the roots of

$$
p_{1}(z) \equiv\left(2 z+\gamma_{1} \tau\right) \cosh z-\left(\gamma_{1} \tau\right) \sinh z .
$$




\section{ONE-DIMENSIONAL DISTORTIONLESS PROPAGATION}

Such quasi-polynomials were considered in the memoir [6] by extending the Sturm method. A different approach would be the use of the Liapunov functional

$$
V(\phi)=\frac{1}{2}(\phi(0)+\phi(-2 \tau))^{2}+\beta \int_{-2 \tau}^{0} \phi^{2}(\sigma) \mathrm{d} \sigma .
$$

The Liapunov approach is important also for the control problem - synthesis of the feedback control $u(t)$ in order to stabilize the system.

The discussion of (38) is far more interesting since it is a nonlinear system. Some of the properties were established in [17]; we recall them in brief. The first one deals with the physical significance of the state variables that have a definite sign; mathematically speaking, an invariant set has to be pointed out.

Proposition 1. Consider system (38) under the standard assumptions induced by physics: $\rho>0, \beta_{i}>0, \sigma_{i}>0, \varphi(\eta) \geq 0,0 \leq \eta \leq h$. Then, if $n_{0} \geq 0$, $q_{i}^{0}(\eta) \geq 0,0 \leq \eta \leq h, i=1, \ldots, m$, then $n(t)>0, c_{i}(\eta, t)>0, \forall t>t_{0}$, $0 \leq \eta \leq h, i=1, \ldots, m$.

For stability of the equilibria of (38), it is necessary to compute the equilibria - a fixed $\rho$ in (38) would imply a unique equilibrium. If the system in deviations is written, we obtain a system that may be given in the form

$$
\begin{aligned}
\dot{\zeta}= & -\left(c^{*} x+\alpha \zeta\right)(1+\zeta)+\sum_{1}^{m} \beta_{i} \mathrm{e}^{-\sigma_{i} t} \int_{0}^{h} \varphi(t+\lambda) \gamma_{i}(\lambda) \mathrm{d} \lambda \\
& -\sum_{1}^{m} \beta_{i} \xi_{i}\left[\zeta(t)-\frac{\sigma_{i}}{\xi_{i}} \int_{0}^{t}\left(\int_{0}^{h} \varphi(\eta) \varphi(\eta+\theta-t) \mathrm{d} \eta\right) \mathrm{e}^{-\sigma_{i}(t-\theta)} \zeta(t+\theta) \mathrm{d} \theta\right] .
\end{aligned}
$$

This structure is obtained by writing the formula of the variation of parameters for the difference equation in (38) and substituting it in the equation for $\zeta$ - the deviation of $n$ with respect to the equilibrium. We have to add the differential equation for the external circuits of the reactor, which defines the state vector $x$,

$$
\dot{x}=A x+b \zeta \text {. }
$$

With a very non-rigorous motivation this model is replaced by the following

$$
\begin{aligned}
\dot{x}= & A x+b \zeta, \\
\dot{\zeta}= & -\left(c^{*} x+\alpha \zeta\right)(1+\zeta) \\
& -\sum_{1}^{m} \beta_{i} \xi_{i}\left[\zeta(t)-\frac{\sigma_{i}}{\xi_{i}} \int_{-\infty}^{t}\left(\int_{0}^{h} \varphi(\eta) \varphi(\eta+\theta-t) \mathrm{d} \eta\right) \mathrm{e}^{-\sigma_{i}(t-\theta)} \zeta(t+\theta) \mathrm{d} \theta\right]
\end{aligned}
$$




\section{VLADIMIR RĂSVAN}

which is very convenient for the construction of a suitable Liapunov functional [13, 17. Therefore the last model (45) is indeed useful and it is a sound mathematical challenge to explain it rigorously. If we consider the integro-differential equation for $\zeta$, it is easy to observe that the two models are equivalent provided for each $i$ and each $\gamma_{i}(\lambda), 0 \leq \lambda \leq h$, it is possible to find some $\zeta_{i}(\lambda)$, $-\infty<\lambda<0$, such that the following holds:

$$
\sigma_{i} \int_{-\infty}^{0}\left(\int_{0}^{h} \varphi(\eta) \varphi(\eta+\theta-t) \mathrm{d} \eta\right) \mathrm{e}^{\sigma_{i} \theta} \zeta(t+\theta) \mathrm{d} \theta=\int_{0}^{h} \varphi(t+\lambda) \gamma_{i}(\lambda) \mathrm{d} \lambda .
$$

If the book 15] is to be reminded, namely its chapter dealing with integral blocks, the above integral equation defines a controllability problem; it is an equation of the first kind and its solution should allow a convenient (from the stability point of view) dependence of $\zeta_{i}(\cdot)$ and $\gamma_{i}(\cdot)$. This is still an open problem, but it is felt it could be solved within the framework of the standard theory for linear integral equations. Indeed, a simple and quite straightforward manipulation of (46) Shows that $\zeta_{i}(\cdot)$ may be considered $h$-periodic and determined on $(-h, 0)$ from the following Fredholm equation of the first kind

$$
\int_{0}^{h} \mathrm{e}^{-\sigma_{i} \theta} \varphi(-\lambda-\theta) \zeta_{i}(-\theta) \mathrm{d} \theta=\left(1-\mathrm{e}^{-h \sigma_{i}}\right) \gamma_{i}(h-\lambda), \quad 0<\lambda<h .
$$

\section{Boundary control of the hyperbolic system of conservation laws}

This paragraph has been stimulated by the problems in [8]. Generally speaking, many of the models in process control arise from the conservation laws: we think, first of all, those models described by ODE. If one-dimensional propagation is considered, the system of the conservation laws accounts for standard models of systems containing steam, water, gas pipes or electrical transmission lines. Especially equations arising from fluid mechanics (unlike those from electrical engineering - see the first section) are nonlinear and, therefore, it is possible to write down only a representation of the form

$$
\frac{\partial Y}{\partial t}+\frac{\partial}{\partial \lambda} f(Y)=0, \quad t>0, \quad 0 \leq \lambda \leq L
$$

with $f: \Omega \subseteq \mathbb{R}^{2} \mapsto \mathbb{R}^{2}$ being the so-called flux density. To this we may add the two boundary conditions

$$
g_{0}\left(Y(0, t), u_{0}(t)\right)=0, \quad g_{L}\left(Y(L, t), u_{L}(t)\right)=0,
$$




\section{ONE-DIMENSIONAL DISTORTIONLESS PROPAGATION}

$u_{0}$ and $u_{L}$ being the control actions. The novelty of this model for control is the nonlinear structure of (48) unlike the previous cases where the PDE are supposed linear; but the models there had as counterpart a control dynamics at the boundaries. The approach consists of the exact diagonalizing of (48), i.e., a correct introduction of the Riemann invariants (the "waves"); the next step is nevertheless linearization (of the diagonal structure) and the choice of a quadratic c.l.f. (Control Liapunov Function(al)). A better approach (in our opinion) would be the use of the energy integral to construct an improved c.l.f.

\section{Conclusions and future research}

The paper contains, in fact, mostly open problems or only solved partly. We reproduce them below:

- stability analysis and stabilization of the overhead crane model;

- complete Liapunov analysis of the circulating fuel nuclear reactor (with possible re-writing of the model);

- boundary control of the system of conservation laws in the nonlinear case (with possible re-writing of previous results concerning steam or water pipe system control).

\section{REFERENCES}

[1] D'ANDRÉA-NOVEL, B.-LEVINE, J.: Modeling and nonlinear control of an overhead crane, in: Proc. of the International Symposium-MTNS '89, Vol. 2, Amsterdam, Netherlands, Jun 19-23, 1989 (M. A. Kaashoek et al., eds.), Progr. Systems Control Theory, Vol. 4, Birkhäuser, Boston, MA, 1990, pp. 523-529.

[2] D'ANDRÉA-NOVEL, B.-BOUSTANY, F.-CONRAD, F.-RAO, B.P.: Feedback stabilization of a hybrid PDE-ODE system: application to an overhead crane, Math. Control Signals Systems 7 (1994), 1-22.

[3] BERNOULLI, J.: Meditationes. De chordis vibrantibus, cum pondusculis aequali intervallo a se invicem dissitis, uni nimirum ex principio virium vivarum quaeritur numerus vibrationum chordae pro una oscillatione penduli datae longitudinis D, Comment. Acad. Sci. Imp. Petropolitanae 3 (1728), 13-28.

[4] BURKE, V.-DUFFIN, R. J.-HAZONY, D.: Distortionless wave propagation in inhomogeneous media and transmission lines, Quart. Appl. Math. XXXIV (1976), 183-194.

[5] CAMMI, A.-DI MARCELLO, V.—LUZZI, L.: Modelling of circulating nuclear fuels with COMSOL multiphysics, in: Proc. of European COMSOL Conference, Vol. 1, Grenoble, France, 23-24 October, 2007, pp. 380-386.

[6] ČEBOTAREV, N. G.-MEİMAN, N. N.: The Routh-Hurwitz Problem for Polynomials and Entire Functions, Tr. Mat. Inst. Steklova, Vol. 26, Nauka, Moscow, 1949. (In Russian) 


\title{
VLADIMIR RĂSVAN
}

[7] COOKE, K. L.: A linear mixed problem with derivative boundary conditions, in: Seminar on Differential Equations and Dynamical Systems (III), Lecture Ser., Vol. 51, Univ. of Maryland, College Park, 1970, pp. 11-17.

[8] CORON, J. M.-D'ANDRÉA-NOVEL, B-BASTIN, G.: A strict Liapunov function for boundary control of hyperbolic systems of conservation laws, IEEE Trans. Automat. Control 52 (2007), 2-11.

[9] COURAnT, R.: Hyperbolic Partial Differential Equations and Applications. in: Modern mathematics for the engineer (E. F. Beckenbach, ed.), McGraw Hill, New York, 1956.

[10] ELSGOLTS, L. E.-NORKIN, S. B.: Introduction to the Theory and Applications of Differential Equations with Deviating Arguments, Nauka, Moscow, 1971 (In Russian), Math. Sci. Engrg., Vol. 105, Academic Press, New York, 1973.

[11] FLIESS, M.-LÉVINE, J.-ROUCHON, P.: A simplified approach of crane control via a generalized state-space model, in: Proc. 30th IEEE Control Decision Conference, Brighton UK, 1991, IEEE, New York, pp. 736-741.

[12] FLIESS, M.-LÉVINE, J.-ROUCHON, P.: Generalized state-variable representation for a simplified crane description, Internat. J. Control 58 (1993), 277-283.

[13] GORJACHENKO, V. D.: Methods for Nuclear Reactors Stability Study. Atomizdat, Moscow, 1977. (In Russian)

[14] PINNEY, E.: Ordinary Difference-Differential Equations. Univ. of California Press, Berkeley, 1958.

[15] POPOV, V. M.: Hyperstability of Control Systems. Editura Academiei, Bucharest, 1966. (In Romanian), (Russian improved version by Nauka Publ. House, Moscow, 1970; French improved version by Dunod, Paris, 1973; English improved version by Springer-Verlag, Berlin, 1973).

[16] RĂSVAN, VL.: Functional differential equations of lossless propagation and almost linear behavior, in: Proc. 6th IFAC Workshop on Time Delay Systems l'Aquila Italy, 2006, 13p. CD-ROM.

[17] RĂSVAN, VL.: Functional differential equations associated to propagation, in: Topics in Time Delay Systems. Analysis, Algorithms and Control (J. J. Loiseau et al., eds.), Lecture Notes in Control and Inform. Sci., Vol. 388, Springer, New York 2009, pp. 293-302,

[18] STAFFAnS, O. J.: A Neutral FDE Is Similar to the Product of an ODE and a Shift. Helsinki Univ. of Technology Inst. of Mathem. Res. Report., A319, April, 1993.

Received September 8, 2008

\author{
Department of Automatic Control \\ Faculty of Automation, \\ Computers and Electronics \\ University of Craiova \\ A. I. Cuza Str. No. 13 \\ RO-200585-Craiova \\ ROMANIA
}

E-mail:vrasvan@automation.ucv.ro 